\title{
UM ÍNDICE DE AVALIAÇÃO DO DESEMPENHO OPERACIONAL E ECONÔMICO-FINANCEIRO DE HOSPITAIS SEM FINS LUCRATIVOS NO BRASIL
}

\section{AN INDEX FOR OPERATIONAL AND ECONOMIC-FINANCIAL PERFORMANCE OF NONPROFIT HOSPITALS IN BRAZIL}

\begin{abstract}
RESUMO
O presente estudo teve por objetivo determinar as variáveis que melhor explicam o desempenho operacional e econômico-financeiro dos hospitais sem fins lucrativos, para com isso, sugerir a construção de um índice de desempenho destas instituições brasileiras. Para tanto, utilizou-se de uma amostra de 63 hospitais sem fins lucrativos, no período de 2006 a 2015 e uma análise fatorial por componentes principais (ACP) com dados em painel não balanceado. Como resultados, obteve-se quatro variáveis econômico-financeiras (Estrutura de Capital/Rentabilidade, Liquidez, Rotatividade e Eficiência dos Ativos) e duas variáveis operacionais (Permanência/Mortalidade e Eficiência Operacional). A variável Estrutura de Capital/Rentabilidade correspondeu a 37,1\% da variância explicada, enquanto as variáveis Liquidez, Rotatividade e Eficiência dos Ativos corresponderam a 23,5\%, 14,5\% e 14,5\%, respectivamente. Já as variáveis Permanência/Mortalidade e Eficiência Operacional explicaram $47,47 \%$ e $44,49 \%$, nessa ordem. Por meio dos índices econômico-financeiro e operacional, observou-se valores abaixo da média em $50 \%$ da amostra, evidenciando que metade das observações exibe desempenho econômico-financeiro e operacional que merecem atenção dos gestores dos hospitais.
\end{abstract}

Palavras-chave: Análise fatorial por componentes principais. Taxa Média de Permanência. Gestão hospitalar.

\begin{abstract}
The present study aimed to determine the variables that best explain the operational and economic-financial performance of non-profit hospitals, in order to suggest the construction of a performance index for these Brazilian institutions. For that, we used a sample of 63 non-profit hospitals from 2006 to 2015 and a factor analysis by main components (PCA) with data in an unbalanced panel. As a result, four economic and financial variables were obtained (Capital Structure/Profitability, Liquidity, Turnover and Efficiency of Assets) and two operational variables (Permanence/Mortality and Operational Efficiency). The variable Capital Structure/Profitability corresponds to $37.1 \%$ of the explained variance, while the variables Liquidity, Turnover and Efficiency of Assets corresponded to $23.5 \%, 14.5 \%$ and $14.5 \%$, respectively. The variables Permanence/Mortality and Operational Efficiency explained $47.47 \%$ and $44.49 \%$, in that order.

In addition, through the economic-financial and operational indices, values below the average were observed in $50 \%$ of the sample, showing that half of the observations show economic-financial and operational performance that deserve attention from hospital managers.
\end{abstract}

Keywords: Analysis by main components. Average Stay Rate. Hospital management.

\begin{abstract}
Déborah Mara Siade Barbosa
Doutora em Administração pela Universidade Federal de Minas Gerais (UFMG). Mestra em Administração pela Universidade Federal de Lavras (UFLA). MBA em Administração de Empresas com ênfase em Finanças pela Fundação Getúlio Vargas (FGV-EAESP). Graduada em Administração pela Universidade Federal de Minas Gerais (UFMG). E-mail: deborahsiade@yahoo.com.br
\end{abstract}

\section{Antônio Artur de Souza \\ Pós-doutor em Finanças pela Universidade de Grenoble. Ph.D. em Management Science pela Universidade de Lancaster. Mestre em Engenharia de Produção pela Universidade Federal de Santa Catarina (UFSC). Graduado em Ciências Contábeis pela Universidade Federal de Santa Catarina (UFSC). Professor Titular do Departamento de Ciências Administrativas da Universidade Federal de Minas Gerais (UFMG). E-mail: artur@face.ufmg.br}

\section{João Eduardo Ribeiro}

Doutorando em Administração no Centro de Pós-Graduação e Pesquisas em Administração (CEPEAD) da Universidade Federal de Minas Gerais (UFMG). Mestre em Administração pelo Centro Federal de Educação Tecnológica de Minas Gerais (CEFET-MG). Graduado em Administração pela Universidade Federal de Minas Gerais (UFMG) e em Ciências Contábeis pela Universidade Federal de Viçosa (UFV). E-mail: joaoribeiro.cco@gmail.com 


\section{INTRODUÇÃO}

Apesar de a Constituição Brasileira de 1988 assegurar que a demanda dos serviços de saúde deve ser atendida, considerando os princípios da universalidade, integralidade e equidade, o que se observa cotidianamente são problemas nevrálgicos e recorrentes, como a concentração de serviços hospitalares em áreas urbanas, a competição entre hospitais e a rede básica de atenção à saúde, insolvência financeira, resolutividade limitada, não consideração do perfil epidemiológico regional entre outros (Ministério da Saúde, 2004). Diante deste panorama, os gestores se deparam com o desafio de coletar e tratar informações, com vistas a alcançar eficácia e eficiência operacional e, consequentemente, a melhoria na gestão hospitalar (Cherchiglia \& Dallari, 2006, Cunha \& Côrrea, 2013).

Para tanto, faz-se adequada a utilização da avaliação de desempenho para aferir os resultados obtidos pelas organizações. Considerando a avaliação de desempenho como uma ferramenta gerencial é possível, por meio dela, verificar o alinhamento entre as estratégias traçadas e os resultados alcançados (Kaplan \& Norton, 2008), bem como, para avaliar a saúde da organização (Neely, 2005) e para aferir os resultados financeiros decorrentes de sua operação. Essas informaç̃̃es são do interesse dos dirigentes, corpo clínico, investidores, benfeitores e os financiadores públicos, ou seja, stakeholders que se interessam em saber os efeitos alcançados com os recursos disponibilizados. Nesse cenário, destaca-se os hospitais sem fins lucrativos que, como a própria denominação expressa, não visam o lucro, no entanto, necessitam cultivar boa saúde financeira, com vistas a se perpetuarem, isto é, precisam de recursos (também financeiros) que garantam suas sustentabilidades ao longo do tempo.

Inicialmente, os estudos que se dedicavam a avaliar o desempenho das organizações hospitalares visavam avaliá-las tendo em vista apenas seu sucesso financeiro (Barbosa, Souza \& Santos, 2015). Essa perspectiva tem evoluído, a fim de contemplar, na avaliação de desempenho, métricas financeiras e não financeiras. Apesar da variedade de indicadores existentes para mensurar a atuação dos hospitais, as críticas estão centradas na inexistência de modelos de avaliação específicos para as organizações hospitalares (Prochnik \& Fonseca, 2006).

Tendo em vista as necessidades de mensuração do desempenho da gestão dos hospitais sem fins lucrativos, a fim de se verificar a utilização dos recursos destinados a tais organizações, questiona-se: Quais as variáveis econômico-financeiras e operacionais que, conjuntamente, auxiliam na construção de um indice de desempenho para hospitais sem fins lucrativos no Brasil? Nesse sentido, o presente estudo tem por objetivo determinar as variáveis que melhor explicam o desempenho operacional e econômico-financeiro dos hospitais sem fins lucrativos para com isso, sugerir a construção de um índice de desempenho destas instituições brasileiras.

Apesar das transformações pelas quais as organizações hospitalares brasileiras têm passado, a insuficiência de recursos, sobretudo financeiros, se mostra notícia recorrente, em se tratando dos hospitais sem fins lucrativos. Assim, a identificação de variáveis econômico-financeiras e operacionais que conduzam a possibilidades de avaliação do desempenho dos hospitais sem fins lucrativos de forma mais refinada, consiste em uma alternativa de ferramental a ser utilizado por vários stakeholders presentes neste contexto. Além disso, a utilização de indicadores econômico-financeiros ou operacionais, isoladamente, não reflete a realidade multidimensional das organizações hospitalares, ensejando a necessidade de avaliar seus desempenhos por meio da junção destes tipos de variáveis.

\section{FUNDAMENTAÇÃO TEÓRICA}

Os hospitais são considerados filantrópicos, caso atendam às disposições estabelecidas na Lei 12.101 de 2009 e obtenham o Certificado de Entidade Beneficente de Assistência Social (CEBAS). Lima et al. (2007) destacam a importância dos hospitais filantrópicos para a saúde do país, haja vista corresponderem a 1/3 dos leitos disponíveis no Brasil e consistirem em uma rede pulverizada por todo o território nacional, com número relevante de pequenos hospitais de baixa complexidade presentes em pequenos municípios e, na metade desses, sendo o único hospital da cidade. Também se mostram importantes por incluírem alguns hospitais de alta complexidade, usualmente, localizados em regiões metropolitanas. No entanto, os autores afirmam que, apesar da importância dessas organizações hospitalares, elas inspiram cuidados e atenção, pois suas estruturas e processos gerenciais podem ser classificados como ausentes ou incipientes.

Neste sentido, a aferição do desempenho desses hospitais, com vistas a verificar a eficiência de suas operações se apresenta como um desafio. Ademais, como se trata de organizações sem fins lucrativos, a importância dada à mensuração do desempenho da organização tende a ser menor, quando comparada às organizações particulares, que buscam a eficiência e elevados índices de desempenho, com vistas a alcançar o maior lucro possível (Huerta, Ford, Peterson \& Brigham, 2008).

No Brasil, a utilização de indicadores de desempenho dos hospitais foi deflagrada pelas definições oriundas do processo de acreditação hospitalar (Schirsari \& Kisil, 2003). Esse processo, realizado pelo Ministério da Saúde, sofreu duras críticas por ser um instrumento baseado em uma avaliação do tipo "possui" ou "não possui", não considerando a organização de saúde de forma integrada e de maneira ampla. Além disso, este tipo de avaliação não contribui para o desenvolvimento de melhorias, pois não há espaço para questionamentos nem para a proposição de soluções (Erdmann \& Moreira, 2010).

Indicadores operacionais e financeiros estão relacionados quando se trata de desempenho em hospitais, afinal, trata-se de ambientes complexos com procedimentos, usualmente, de custos elevados. Para ofertar serviços de qualidade à população é importante que os hospitais sejam financeiramente saudáveis (Lima Neto, 2011). Logo, a utilização de 
indicadores econômico-financeiros se faz importante para analisar o desempenho dos hospitais (Watkins, 2000; Tavares \& Silva, 2012). O desempenho organizacional também pode ser considerado uma medida de eficiência com que os hospitais se utilizam dos recursos de que dispõem para prestar os serviços que lhe são característicos, quando comparados a outros hospitais (Barbosa et al., 2015).

Souza, Rodrigues, Lara, Guerra e Pereira (2009) defendem que os indicadores de desempenho econômico-financeiro possuem como função avaliar a situação econômico-financeira das organizações hospitalares, visando quantificar os resultados e avaliar o cumprimento de objetivos e metas, demonstrando a situação econômico-financeira dessas organizações.

Outrossim, o desempenho operacional pode ser aferido por medidas estabelecidas para avaliar as operações das organizações (Perera, Harrison \& Poole, 1997). Barbosa et al. (2015) afirmam que as variáveis operacionais mais utilizadas nos estudos que avaliam o desempenho hospitalar estão relacionadas a leitos hospitalares, equipe médica hospitalar, equipe de enfermagem (enfermeiros e técnicos de enfermagem), centros cirúrgicos, internações, atendimentos emergenciais e intervenções cirúrgicas.

Apesar da dissonância existente na literatura acerca dos indicadores mais adequados para aferir o desempenho das organizações hospitalares, Barnum e Kutzin (1993) apontam a Taxa Média de Permanência (TMP), a Taxa de Ocupação dos Leitos (TO) e a Taxa de Rotatividade (TR) como indicadores úteis para esclarecer a variação dos custos operacionais. Segundo os autores, considerando o perfil de custo do tratamento como similar para diferentes tipos de hospitais, uma TO mais elevada tende a resultar em custos, por paciente, mais baixos, haja vista, os custos indiretos serem distribuídos por leitos ocupados. No que tange a TO e à taxa de rotatividade, pode-se dizer que quanto maior esses indicadores, maior a eficiência do hospital, visto que indicam baixa ociosidade dos leitos. Por outro lado, a TO elevada pode indicar baixa resolutividade e incidência de moléstias crônicas, resultando em pouca reserva de leitos para eventuais emergências. Além disso, elevadas taxas de rotatividade podem indicar relevante quantidade de reinternações.

O reconhecimento da precisão de indicadores específicos para esse setor, dado a suas peculiaridades (Côrrea \& Hourneaux Jr., 2008) e a ciência das discrepâncias causadas na avaliação de desempenho dos mesmos, em decorrência de suas especificidades, se apresenta como avanço tímido, mas necessário à evolução do processo de avaliação de desempenho. A discussão acerca da necessidade dos indicadores já se apresenta como sinal de progresso, sobretudo, por tratar-se de um país emergente (Peckan, Atan \& Beylik, 2011).

Ramos, Parizotto, Silva, Ramos e Bampi (2018) analisaram a relação entre os indicadores econômicos-financeiros e os índices de qualidade hospitalar em hospitais do terceiro setor do sul do país. Os autores demonstraram que, quanto mais as entidades hospitalares prestarem um serviço de qualidade, maior será o retorno financeiro e, portanto, maiores serão os recursos para investimentos.

Em linha com o estudo de Ramos et al. (2018), Silva, Sell e Ferla (2018) analisaram a relação entre as características organizacionais e o desempenho econômico-financeiro de hospitais sem fins lucrativos. Como resultados, os autores apontam que: (i) quanto mais leitos os hospitais possuem maior é a utilização de recursos de terceiros; (ii) a liquidez dos hospitais filantrópicos da região sul reduz na medida em que se eleva o total dos ativos e (iii) priorizar procedimentos de alta complexidade não contribui para o desempenho positivo dos hospitais.

Ainda no setor da saúde, Xavier, Souza e Avelar (2019) analisaram o desempenho econômico-financeiro das Operadoras de Planos de Saúde (OPS) brasileiras, no período entre 2010 e 2015 e evidenciaram que, dentre os grupos de indicadores analisados, os de rentabilidade/lucratividade apresentaram a maior variabilidade na amostra analisada, demonstrando que a gestão empresarial pode ser fator decisivo para melhores resultados em termos de rentabilidade e lucratividade.

\section{PROCEDIMENTOS METODOLÓGICOS}

\subsection{Amostra}

A presente pesquisa, classificada como descritiva, quantitativa e ex-post fact (Hair, Black, Babin, Anderson \& Tatham, 2009) teve como amostra 63 hospitais sem fins lucrativos, escolhidos conforme a disponibilidade de suas demonstrações contábeis - Balanço Patrimonial (BP) e Demonstração do Resultado do Exercício (DRE) e informações operacionais.

A pesquisa completa será executada em duas fases. Este primeiro estudo contemplou a primeira fase, com dados de 2006 a 2015, na qual foram apontadas as variáveis que melhor explicam o desempenho operacional e econômico-financeiro dos hospitais sem fins lucrativos, para com isso, construir um índice de desempenho destas instituições brasileiras. Já a segunda fase, em andamento, contempla dados de 2010 até 2020, com os quais será testado novamente o desempenho do índice criado nesta primeira fase. Nesta segunda fase, se espera um corpus de igual tamanho para confrontar os resultados. Os dados foram coletados via DATASUS, SIHSUS e CNES - bancos de dados importantes sobre a saúde no Brasil (Brasil, 2009) - e; tabulados, compilados e analisados utilizando-se os softwares Excel e SPSS 17.0. A Tabela 1 traz a amostra completa, identificado por meio do número do Cadastro Nacional de Estabelecimentos de Saúde (CNES). 
Tabela 1 - Amostra do Estudo

\begin{tabular}{c|c|c|c|c|c|c|c|c|c}
\hline $\mathbf{n}^{\mathbf{0}}$ & CNES & $\mathbf{n}^{\mathbf{0}}$ & CNES & $\mathbf{n}^{\mathbf{0}}$ & CNES & $\mathbf{n}^{\mathbf{0}}$ & CNES & $\mathbf{n}^{\mathbf{0}}$ & CNES \\
\hline 1 & 2007037 & 14 & 0009733 & 27 & 2537788 & 40 & 2079097 & 53 & 2079461 \\
2 & 2010151 & 15 & 2371375 & 28 & 2379333 & 41 & 2027186 & 54 & 2079135 \\
3 & 2018098 & 16 & 2756951 & 29 & 2662914 & 42 & 2082853 & 55 & 2083116 \\
4 & 0003808 & 17 & 2397684 & 30 & 2754843 & 43 & 2079461 & 56 & 2791749 \\
5 & 2651394 & 18 & 2592746 & 31 & 2078252 & 44 & 2079135 & 57 & 2081350 \\
6 & 0011746 & 19 & 2354551 & 32 & 2083116 & 45 & 2081105 & 58 & 2080532 \\
7 & 0011738 & 20 & 2344882 & 33 & 2079461 & 46 & 2082624 & 59 & 2080796 \\
8 & 2456958 & 21 & 0015644 & 34 & 2079135 & 47 & 2751623 & 60 & 2077531 \\
9 & 2165058 & 22 & 2409151 & 35 & 2081105 & 48 & 2089572 & 61 & 2058391 \\
10 & 0026808 & 23 & 2232928 & 36 & 2082624 & 49 & 2747871 & 62 & 2077477 \\
11 & 2219638 & 24 & 2241072 & 37 & 2751623 & 50 & 2079097 & & \\
12 & 2695375 & 25 & 3006522 & 38 & 2089572 & 51 & 2027186 & 63 & 2080575 \\
13 & 2148293 & 26 & 2558017 & 39 & 2747871 & 52 & 2082853 &
\end{tabular}

Fonte: Elaborada pelos autores.

\subsection{Variáveis}

A seleção das variáveis econômico-financeiras e operacionais baseou-se na revisão de literatura nacional e internacional acerca da temática desempenho de organizações hospitalares. A Tabela 2 exibe as variáveis econômico-financeiras utilizadas, separados por grupos de análise: (1) liquidez, (2) rotatividade, (3) eficiência dos ativos, (4) lucratividade, (5) endividamento e estrutura de capital e (6) rentabilidade, conforme listado a seguir.

Tabela 2 - Variáveis Econômico-financeiras

\begin{tabular}{|c|c|c|c|}
\hline & Indicadores & Fórmulas & Trabalhos \\
\hline : & $\begin{array}{c}\text { Liquidez Geral (LG) } \\
\text { Liquidez Corrente (LC) } \\
\text { Liquidez Seca (LS) }\end{array}$ & $\begin{array}{c}((A C+R L P)) /((P C+P N C)) \\
A C / P C \\
((A C-E \text { stoques })) / P C\end{array}$ & $\begin{array}{l}\text { Faria, Azevedo \& Oliveira (2012), } \\
\text { Souza et al. (2009), Zeller et al. } \\
\text { (1996). }\end{array}$ \\
\hline 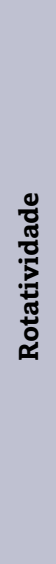 & $\begin{array}{l}\text { Prazo Médio de Recebimento (PMR) } \\
\text { Prazo Médio de Estocagem (PME) } \\
\text { Prazo Médio de Pagamento (PMP) } \\
\text { Ciclo Operacional (CO) } \\
\text { Ciclo Financeiro (CF) } \\
\text { Giro dos Estoques (GE) } \\
\text { Giro de Contas a Receber (GCR) }\end{array}$ & $\begin{array}{c}\frac{\text { Contas a Receber }}{\text { Receita Bruta }} \times 360 \text { dias } \\
\frac{\text { Estoque Médio }}{\text { Custo dos Serviços Prestados }} \times 360 \text { dias } \\
\frac{\text { Fornecedores }}{\text { Compras de Materiais e Serviços }} \times 360 \text { dias } \\
\text { PMR/PME } \\
\text { PMP+CO } \\
\text { (Custo )/(Estoque Médio) } \\
\text { (Receitas Operacionais)/ } \\
\text { (Contas a Receber) }\end{array}$ & $\begin{array}{l}\text { Souza et al. (2009), Guerra e Souza } \\
\text { (2011). }\end{array}$ \\
\hline
\end{tabular}




\begin{tabular}{|c|c|c|c|}
\hline 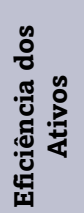 & $\begin{array}{c}\text { Giro do Ativo (GA) } \\
\text { Giro do Ativo Fixo (GAP) } \\
\text { Giro do Ativo Circulante (GAC) }\end{array}$ & $\begin{array}{c}R O L / A T \\
R O L /((A N C-R L P)) \\
R O L / A C\end{array}$ & $\begin{array}{c}\text { Souza et al. (2009), Zeller et al. } \\
\text { (1996), Watkins (2000), Silva (2014), } \\
\text { Barnum e Kutzin (1993). }\end{array}$ \\
\hline 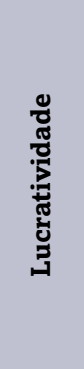 & $\begin{array}{c}\text { Margem Líquida (ML) } \\
\text { Margem Operacional (MO) } \\
\text { Margem Receita Não Operacional (MRNO) } \\
\text { Margem Bruta (MB) }\end{array}$ & $\begin{array}{l}\text { (Superávit ou déficit Liquido)/ } \\
\qquad R O L \\
\text { (Superávit ou déficit } \\
\text { Operacional)/ROL } \\
R N O /(R O L) \\
\text { (Superávit ou déficit Bruto)/ } \\
R O B\end{array}$ & $\begin{array}{l}\text { Souza et al. (2009), Guerra e Souza } \\
\text { (2011), Silva (2014), Veloso e Malik } \\
\text { (2010), Barnum e Kutzin (1993). }\end{array}$ \\
\hline 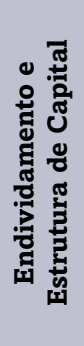 & $\begin{array}{l}\text { Imobilização do Patrimônio Líquido (IPL) } \\
\text { Imobilização dos Recursos não Correntes } \\
\text { (IRNC) } \\
\text { Composição do Endividamento (CE) } \\
\text { Participação de Capital de Terceiros (PCT) }\end{array}$ & $\begin{array}{c}A P / P L \times 100 \\
A P /((P L+E L P)) \times 100 \\
A P /((P L+E L P)) \times 100 \\
((P C+E L P)) /(P L) \times 100\end{array}$ & $\begin{array}{c}\text { Souza et al. (2009), Zeller et al. } \\
\text { (1996), Watkins (2000), Silva (2014), } \\
\text { Veloso e Malik (2010). }\end{array}$ \\
\hline 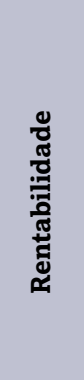 & $\begin{array}{c}\text { Margem EBITDA } \\
\text { Margem EBIT } \\
\text { Retorno sobre o Capital Investido (ROIC) } \\
\text { Retorno sobre o Patrimônio Líquido (ROE) } \\
\text { Retorno sobre o Ativo (ROA) }\end{array}$ & $\begin{array}{c}((P C+E L P)) /(P L) \times 100 \\
((P C+E L P)) /(P L) \times 100 \\
L O / C I \times 100 \\
L L /(P L) \quad X 100 \\
L L /(A T) \quad X 100\end{array}$ & $\begin{array}{c}\text { Zeller et al. (1996), Watkins (2000), } \\
\text { Silva (2014), Veloso e Malik (2010), } \\
\text { Souza et al. (2009). }\end{array}$ \\
\hline
\end{tabular}

Nota: Ativo Circulante (AC), Realizável a Longo Prazo (RLP), Passivo Circulante (PC), Passivo não Circulante (PNC), Receita Operacional Líquida (ROL), Ativo Total (AT), Ativo não Circulante (ANC), Receita Não Operacional (RNO), Receita Operacional Bruta (ROB), Ativo Permanente (AP), Patrimônio Líquido (PL), Exigível a Longo Prazo (ELP), Receita Líquida (RL), Lucro Operacional (LO), Capital Investido (CI), Lucro Líquido (LL).

Fonte: Elaborada pelos autores.

Além destes indicadores, outros também são importantes para evidenciar a estrutura de capital e de dívidas dos hospitais, bem como a rentabilidade dos mesmos. Dentre esses indicadores, têm-se: (i) a relação Ativo total/Patrimônio líquido; (ii) o Passivo não circulante em relação ao passivo e PL; (iii) o Passivo não circulante/Patrimônio líquido; (iv) a Dívida líquida/EBITDA; (v) a Dívida líquida/Imobilizado líquido; (vi) as Despesas financeiras/Dívida líquida e (vii) a Dívida líquida/PL (Iudícibus, 2017).

No tocante às outras relações importantes para evidenciar a rentabilidade dos hospitais têm-se: (i) o Custo/receita líquida; (ii) o SG\&A/RL (selling, general and administrative expenses) ou despesas de vendas, gerais e administrativas em relação à receita líquida de serviços e (iii) as Despesas financeiras/Receita líquida (Iudícibus, 2017).

No que concerne às variáveis operacionais, a Tabela 3 as apresenta, juntamente com os trabalhos que as utilizaram anteriormente e as fórmulas de cálculo. A TMP tem por objetivo demonstrar o prazo médio de permanência dos pacientes internados. Por sua vez, a TO indica a capacidade ocupada do hospital. 
Tabela 3 - Variáveis Operacionais

\begin{tabular}{|c|c|c|}
\hline Indicador & Fórmulas & Trabalhos \\
\hline \multirow{2}{*}{ Taxa Média de Permanência (TMP) } & Total dos pacientes internados no período & \multirow{12}{*}{$\begin{array}{l}\text { Marinho, Moreno e Cavalini } \\
\text { (2001), La Forgia e Coutto- } \\
\text { lenc (2009), Vecina Neto e } \\
\text { Malik (2011), Ramos et al. } \\
\text { (2015), Marinho e Façanha } \\
\text { (2001), Silva, Barreto, } \\
\text { Souza e Lucena (2006), Silva } \\
\text { (2008), Souza et al. (2009), } \\
\text { Guerra e } 2009 \text { (2011). }\end{array}$} \\
\hline & Total de pacientes com alta no período $X 100$ & \\
\hline \multirow{2}{*}{ Taxa de Ocupação Hospitalar (TO) } & Número de pacientes no período & \\
\hline & Número de leitos no período $X 100$ & \\
\hline \multirow{2}{*}{ Taxa de Rotatividade (TR) } & Número de saídas por alta ou óbito no período & \\
\hline & Número de leitos no período & \\
\hline \multirow{2}{*}{ Taxa de Mortalidade (TM) } & Número de óbitos no período & \\
\hline & Número de saídas por alta ou óbito no período & \\
\hline \multirow{2}{*}{ Número de Profissionais por Leito (NPL) } & Número total de funcionários & \\
\hline & Número total de leitos & \\
\hline \multirow{2}{*}{ Valor Médio de AIH Paga (VAIH) } & Faturamento proveniente das AIH & \\
\hline & $\overline{\text { Quantidade de internações realizadas }}$ & \\
\hline
\end{tabular}

Fonte: Elaborada pelos autores.

Já a TR representa a utilização da capacidade instalada do hospital, desse modo, representa um dos principais indicadores de produtividade hospitalar sob a ótica da eficiência. Assim, quanto maior, melhor. Outro indicador operacional, a Taxa de mortalidade denota a quantidade de óbitos em relação à quantidade de saídas do hospital, seja por motivo de alta ou de óbito, durante o período analisado. O Número de profissionais por leito demonstra a quantidade de profissionais envolvidos no cuidado aos pacientes. Por sua vez, o Valor médio de AIH (Autorização de Internação Hospitalar) paga indica o valor médio de cada internação no período. Este indicador é significativo para a avaliação do faturamento do hospital, apesar de não refletir, de modo pleno, a cobertura dos custos incorridos em cada internação. Finalmente, a Quantidade de leitos disponível indica o número de leitos disponíveis para atendimento aos pacientes. A utilização desta métrica é importante para verificar a relação entre o desempenho dos hospitais e a quantidade de leitos que disponibilizam (La Forgia \& Coutollenc, 2009).

\subsection{Análise fatorial por componentes principais}

Os dados foram tabulados, compilados e analisados utilizando-se os softwares Microsoft $®$ Excel $® 2016$, SPSS $®$ 17.0, Stata ${ }^{\circledR} 14$ e TabWin. Para a determinação das variáveis operacionais e econômico-financeiros que melhor explicam o desempenho dos hospitais sem fins lucrativos utilizou-se a análise fatorial por componentes principais (ACP) com dados em painel não balanceado. A ACP tem como objetivo a adequação de um conjunto de variáveis em um novo conjunto de variáveis, de mesma dimensão, os componentes principais, que captem o comportamento das variáveis iniciais (Fávero \& Belfiore, 2015). A técnica de ACP se mostra adequada para a criação de índices, conforme demonstra o estudo de Silva, Silva e Borges (2015).

A fim de se garantir a adequabilidade da aplicação da ACP, foram aplicadas as medidas Kaiser-Meyer-Olkin (KMO) e o Teste de esfericidade de Barllet. O KMO varia de 0 a 1 e quanto mais próximo deste último, mais adequada é a aplicação da análise. Malhotra (2006) afirma que resultados do KMO entre 0,5 e 1 já atestam a adequação da análise aos dados. O teste de esfericidade de Barllet é utilizado para averiguar se as correlações de Pearson entre os pares de variáveis são estatisticamente diferentes de zero, atestando que a utilização da análise fatorial se faz adequada e, portanto, as variáveis poderão ser extraídas tendo como base as variáveis originais. Este, deve possuir significância inferior a 1\% denotando que a matriz de correlação dos dados não é uma matriz identidade o que impediria a utilização da análise. O resultado do teste de esfericidade de Barlett deve ser privilegiado quando comparado à estatística KMO no que tange à decisão sobre a adequação global da análise fatorial (Hair et al., 2009).

No intuito de verificar a qualidade da análise, utilizou-se a variância explicada, que deve possuir valores de pelo menos $50 \%$ e as cargas fatoriais das variáveis participantes da análise. A variância explicada revela o quanto as variáveis são capazes de explicar o fator. Em seguida, é preciso observar a comunalidade que revela a quantidade total de variância que uma variável compartilha com as demais participantes da análise. Para serem consideradas adequadas precisam ter valores superiores a 0,4 bem como a carga fatorial de cada variável. Já a avaliação das cargas fatoriais, das variáveis participantes, se faz necessária pelo fato das cargas com valores aproximados (com diferenças entre as cargas inferiores a 0,100 ) em mais de um fator não serem consideradas adequadas, segundo os parâmetros de Hair et al. (2009). 


\subsection{Criação do índice}

Para a construção do índice de desempenho dos hospitais sem fins lucrativos, efetuou-se a análise dos componentes principais de todos os indicadores representativos das dimensões econômico-financeira e operacional. Após a obtenção dos componentes principais econômico-financeiros e operacionais, calculou-se os respectivos índices, por meio do cálculo da média ponderada de todos os componentes principais, sendo os pesos representados pelas respectivas variâncias (Nagar \& Basu, 2002; Fávero \& Belfiore, 2015).

$$
\text { Índice }_{x}=\frac{\sum_{i=1}^{x}(V E * S)}{\sum_{i=1}^{x}(V E)}
$$

Em que: $V E$ é a variância explicada da variável, $S$ é o escore da variável e $x$ é o número de variáveis que compõem o índice.

Optou-se pela utilização da análise de componentes principais, pois nela toda a variância é considerada, tanto a compartilhada quanto a exclusiva, pressupondo-se a inexistência de erro (Dancey \& Reidy, 2006; Francisco, 2014) e por sua utilização em estudos relacioandos à criação de índices no campo das ciências sociais aplicadas (Francisco, 2014; Correia, 2008; Silva, Silva \& Borges, 2015; Krubusly, 2001; Krishnakumar \& Nagar, 2008; Figueiredo Filho, Paranhos, Rocha, Silva \& Maia, 2013).

\section{ANÁLISE E INTERPRETAÇÃO DOS RESULTADOS}

\subsection{Análise descritiva}

A Tabela 4 expõe as medidas de tendência central e dispersão das variáveis econômico-financeiras. Observa-se que os índices de liquidez tiveram média entre 1,14 e 1,53, com a liquidez geral se mantendo inferior aos outros dois indicadores deste grupo. Nota-se que há problemas de liquidez (indicador menor que 1) quando se analisa os valores mínimos encontrados. Uma das causas que justifique a liquidez seca e corrente apresentarem resultados superiores aos da liquidez geral podem estar relacionados às próprias características do negócio, tais como o investimento em máquinas e equipamentos de elevado valor com pagamentos em longo prazo. No tocante aos indicadores de rotatividade, verifica-se que os serviços prestados são pagos sempre com prazo, concedido pelos hospitais, evidenciando que a prestação de serviços para pagamento a prazo possa ser uma característica do setor. É possível notar que o Prazo Médio de Recebimento e o Ciclo Operacional possuem $50 \%$ das observações situadas abaixo da mediana. Já a média do giro de contas a receber apresenta forte oscilação (DP $=81,94)$.

Os indicadores de eficiência dos ativos apresentam certa uniformidade ao longo dos anos, nas variáveis giro do ativo e giro do ativo fixo (não circulante), conforme observado nos baixos valores de desvio-padrão. É importante observar que, na média, os hospitais analisados utilizam melhor os ativos que possuem maior liquidez para a geração de receitas operacionais líquidas do que os ativos fixos, de baixa liquidez. Apesar da variação, os três indicadores se apresentam como satisfatórios do ponto de vista de eficiência dos ativos. Quanto aos indicadores de lucratividade, são uteis, no caso dos hospitais sem fins lucrativos, para verificar a existência de uma situação superavitária. Os indicadores margem operacional e margem líquida têm comportamento negativo, sugerindo que os hospitais não demonstram eficiência operacional, quando considerados de modo agregado.

Pela análise dos indicadores de rentabilidade, observa-se que a situação dos hospitais analisados não é confortável. A margem operacional, por exemplo, apresenta valores abaixo da média em grande parte das observações. Porém, na média, os hospitais se mostram eficientes, ao demonstrarem taxas crescentes de lucros, ao longo do período analisado, conforme evidenciado pela evolução da margem bruta. Diferentemente, as margens operacional e líquida se mostram, na média, próximas a zero ou negativas, denotando que as estruturas operacionais parecem sobrecarregar os hospitais a ponto de neutralizar as receitas operacionais dos mesmos.

Os indicadores de rentabilidade também são afetados pela influência de dados discrepantes, sobretudo, as variáveis que possuem a margem EBITDA em sua composição. Esses indicadores, na média, demonstram que a situação dos hospitais não é confortável. O ROE apresenta uma média de -0,23 e desvio padrão elevado (5,21), demonstrando a elevada variação dos valores observados em relação à média do grupo. Considerando que o ROE e o ROA contemplam o lucro líquido após o imposto de renda como numerador e o ROA se manteve praticamente estável, ao longo do período analisado, é possível que tenha havido brusca queda no PL de algum(ns) hospital(is). 
Tabela 4 - Medidas de tendência central e dispersão das variáveis econômico-financeiras

\begin{tabular}{|c|c|c|c|c|c|c|}
\hline Indicadores & $\begin{array}{c}\mathbf{N} \\
\text { Valid }\end{array}$ & Mínimo & Mediana & Máximo & Média & DP \\
\hline LG & 260 & $-2,27$ & 0,67 & 7,97 & 1,14 & 1,43 \\
\hline $\mathrm{LC}$ & 260 & $-2,83$ & 1,03 & 9,54 & 1,53 & 1,66 \\
\hline LS & 260 & $-2,89$ & 0,93 & 9,28 & 1,41 & 1,61 \\
\hline PMR & 239 & $-646,91$ & 52,04 & 483,47 & 57,86 & 71,98 \\
\hline $\mathrm{CO}$ & 247 & $-683,51$ & 47,28 & 483,47 & 55,64 & 92,59 \\
\hline $\mathrm{CF}$ & 252 & $-683,51$ & 37,91 & 483,47 & 31,62 & 98,53 \\
\hline GCR & 239 & $-965,32$ & 6,54 & 69,52 & $-2,21$ & 81,94 \\
\hline GA & 260 & $-0,40$ & 1,07 & 6,49 & 1,32 & 1,05 \\
\hline GAP & 260 & $-0,47$ & 1,66 & 20,98 & 2,55 & 2,76 \\
\hline GAC & 260 & $-2,57$ & 3,07 & 62,10 & 4,48 & 6,34 \\
\hline ML & 260 & $-2,16$ & 0,00 & 0,76 & $-0,04$ & 0,30 \\
\hline MO & 260 & $-3,30$ & $-0,02$ & 0,83 & $-0,15$ & 0,51 \\
\hline MB & 260 & $-2,24$ & 1,00 & 5,35 & 0,96 & 1,17 \\
\hline IPL & 260 & $-506,48$ & 0,97 & 85,62 & $-0,25$ & 33,38 \\
\hline IRNC & 260 & $-5,89$ & 0,94 & 49,05 & 1,30 & 3,81 \\
\hline $\mathrm{CE}$ & 260 & $-0,72$ & 0,66 & 1,00 & 0,66 & 0,23 \\
\hline PCT & 260 & $-753,72$ & 0,43 & 127,00 & $-1,27$ & 49,61 \\
\hline $\mathrm{AT} / \mathrm{PL}$ & 260 & $-752,72$ & 1,43 & 128,00 & $-0,27$ & 49,65 \\
\hline PNC/PL & 235 & $-511,63$ & 0,11 & 74,28 & $-1,21$ & 34,89 \\
\hline $\begin{array}{c}\text { PNC/ } \\
\text { Passivo+PL }\end{array}$ & 235 & $-0,06$ & 0,19 & 2,56 & 0,33 & 0,37 \\
\hline DL/ EBITDA & 255 & $-778184,78$ & $-0,55$ & 107,84 & $-3056,10$ & 48731,57 \\
\hline $\mathrm{DF} / \mathrm{DL}$ & 242 & $-402,73$ & $-0,11$ & 102,79 & $-1,05$ & 27,42 \\
\hline $\mathrm{DL} / \mathrm{IL}$ & 260 & $-2,48$ & $-0,02$ & 2,44 & $-0,05$ & 0,58 \\
\hline $\mathrm{DL} / \mathrm{PL}$ & 260 & $-40,05$ & $-0,06$ & 78,69 & 0,13 & 6,20 \\
\hline $\begin{array}{l}\text { Margem } \\
\text { EBITDA }\end{array}$ & 255 & $-2,15$ & 0,01 & 4401681,84 & 17261,48 & 275644,01 \\
\hline Margem EBIT & 255 & $-2,15$ & 0,01 & 3417757,40 & 13402,95 & 214028,27 \\
\hline ROIC & 255 & $-2,64$ & 0,01 & 3417757,40 & 13402,93 & 214028,27 \\
\hline $\mathrm{ROE}$ & 260 & $-81,23$ & 0,07 & 10,48 & $-0,23$ & 5,21 \\
\hline ROA & 260 & $-3,99$ & 0,00 & 0,48 & $-0,05$ & 0,33 \\
\hline Custo/RL & 236 & $-3,24$ & $-0,29$ & 4,35 & $-0,02$ & 1,23 \\
\hline SG\&A/RL & 252 & $-2,12$ & $-0,12$ & 1,95 & $-0,23$ & 0,51 \\
\hline $\mathrm{DF} / \mathrm{RL}$ & 242 & $-1,93$ & $-0,01$ & 1,85 & $-0,02$ & 0,18 \\
\hline
\end{tabular}

Nota: Liquidez Geral (LG), Liquidez Corrente (LC), Liquidez Seca (LS), Prazo Médio de Recebimento (PMR), Ciclo Operacional (CO), Ciclo Financeiro (CF), Giro de Contas a Receber (GCR), Giro do Ativo (GA), Giro do Ativo Fixo (GAP), Giro do Ativo Circulante (GAC), Margem Líquida (ML), Margem Operacional (MO), Margem Bruta (MB), Imobilização do Patrimônio Líquido (IPL), Imobilização dos Recursos não Correntes (IRNC), Composição do Endividamento (CE), Participação de Capital de Terceiros (PCT), Ativo Total (AT), Patrimônio Líquido (PL), Passivo não Circulante (PNC), Dívida Líquida (DL), Despesas Financeiras (DF), Imobilizado Líquido (IL), Participação de Capital de Terceiros (PCT), Retorno sobre o Capital Investido (ROIC), Retorno sobre o Patrimônio Líquido (ROE), Retorno sobre o Ativo (ROA), Receita Líquida (RL), Despesas de vendas, gerais e administrativas (SG\&A).

Fonte: Elaborada pelos autores.

No que concerne aos indicadores de endividamento e estrutura de capital, percebe-se que a média das organizações analisadas demonstra dependência preocupante do capital de terceiros para o financiamento do ativo circulante, conforme observado na Tabela 4. Cabe ressaltar que não foi possivel o levantamento dos dados completos para todas as variáveis e, dessa forma, optou-se por excluir da análise, as variáveis prazo médio de estocagem, prazo médio de pagamento, giro dos estoques e margem receita não operacional, por possuírem mais de $75 \%$ de dados ausentes. Além disso, 
realizou-se o teste de Kolmogorov-Smirnov, conforme sugerem Hair et al. (2009), para verificar o padrão de distribuição dos dados e verificou-se que, com exceção das variáveis composição do endividamento, margem bruta, taxa de ocupação e taxa de rotatividade, as demais variáveis em análise não apresenta distribuição normal.

No tocante às variáveis operacionais, apresentadas na Tabela 5, observa-se que, dentre os hospitais da amostra houve, ao longo dos anos observados, decréscimo no número de leitos disponíveis. Por outro lado, o número de profissionais por leito demonstrou elevação média. É possível que a quantidade de profissionais por leito tenha aumentado não em rezão da contratação de pessoal, mas da redução do número de leitos disponíveis. Na visão de Paim, Travassos, Almeida, Bahia \& Macinko (2011) a redução do número de leitos disponível se dá em razão do subfinanciamento operacional pelo qual os hospitais têm passado ao longo dos anos.

Tabela 5 - Medidas de tendência central e dispersão das variáveis operacionais

\begin{tabular}{c|c|c|c|c|c|c}
\hline Variáveis & $\begin{array}{c}\text { N } \\
\text { Valid }\end{array}$ & Mínimo & Mediana & Máximo & Média & DP \\
\hline TMP & 236 & 1,00 & 1,05 & 628,50 & 10,12 & 58,35 \\
TO & 246 & 0,01 & 29,22 & 67,38 & 29,39 & 14,32 \\
TR & 223 & 0,00 & 26,39 & 63,40 & 26,07 & 14,79 \\
TM & 235 & 0,00 & 0,05 & 72,00 & 0,68 & 5,75 \\
VAIH & 260 & 275,08 & 962,00 & 14133,08 & 1529,73 & 1861,65 \\
NPL & 243 & 0,18 & 2,35 & 37,20 & 3,76 & 5,13 \\
Quant. de & 246 & 36,00 & 147,00 & 1172,00 & 218,59 & 208,86 \\
Leitos & & &
\end{tabular}

Nota: Taxa Média de Permanência (TMP), Taxa de Ocupação Hospitalar (TO), Taxa de Rotatividade (TR), Número de Profissionais por Leito (NPL), Valor Médio de AIH Paga (VAIH).

Fonte: Elaborada pelos autores.

Outro indicador operacional, a taxa de mortalidade, sofreu redução durante o período analisado, assim como a taxa de permanência, que também apresentou queda significativa. A redução da taxa de permanência é uma das diretrizes preconizadas pela ANS (2013), que considera o leito um recurso valioso, que deve ser utilizado de forma racional. Além disso, quanto maior o tempo de permanência do paciente no hospital, maior o custo incorrido para tal. As taxas de ocupação e de rotatividade tiveram variações semelhantes. A taxa de ocupação se mantém próximo a um patamar distante do ideal que é de 75 a 85\%, na visão de La Forgia e Coutollenc (2009). Além disso, verifica-se que grande parte das variáveis apresenta elevado desvio-padrão, evidenciando assim, afastamento dos valores observados em relação à média.

\subsection{Análise de Componentes Principais}

Realizou-se a ACP considerando-se as variáveis econômico-financeiras e operacionais. Na primeira análise, apesar das medidas de adequação se mostrarem satisfatórias, foi eliminada a variável imobilização de recursos não correntes, pois a mesma não se agregou a nenhum fator. Nova verificação de adequação aos critérios foi realizada e a variável giro de contas a receber foi eliminada da análise, pois apresentou baixa comunalidade e apresentou cargas fatoriais muito semelhantes em duas variáveis. Na terceira rodada, após satisfeitas as condições de adequação dos dados, foram eliminadas as variáveis PNC/Passivo+PL e SG\&A/RL, pois ambas apresentaram carga similar em mais de um fator e baixa comunalidade. Os mesmos motivos eliminaram a variável ROIC, na quarta rodada e também a variável composição do endividamento, na quinta análise.

$\mathrm{Na}$ sexta rodada, foram eliminadas as variáveis margem líquida, margem operacional, margem bruta, margem EBITDA, margem EBIT e custo/receita líquida, pois todas essas variáveis apresentaram cargas fatoriais com medidas próximas para um mesmo fator. Na sétima rodada foram eliminadas, pelo mesmo motivo, as variáveis giro do ativo circulante e dívida líquida/imobilizado líquido. Na oitava rodada, devido à carga fatorial inferior a 0,4, a variável despesas financeira/dívida líquida também foi excluída. Na nona rodada, a variável ciclo operacional foi eliminada por exibir carga similar em mais de um fator.

Nesse sentido, a Tabela 6 exibe as variáveis econômico-financeiros e operacionais encontrados por meio da ACP, bem como os valores de suas cargas, comunalidades e variâncias explicadas. A verificação da ACP econômico-financeira foi satisfatória, uma vez que obteve KMO igual a 0,718 e significância do teste de esfericidade de Barlett inferior a $1 \%$. Para a análise das variáveis operacionais, o teste de esfericidade de Barllet foi significativo a 1\% e o valor do KMO igual a 0,511, demonstrando a adequação da utilização da técnica para a amostra desta pesquisa. 
Tabela 6 - Resultado da análise de componentes principais

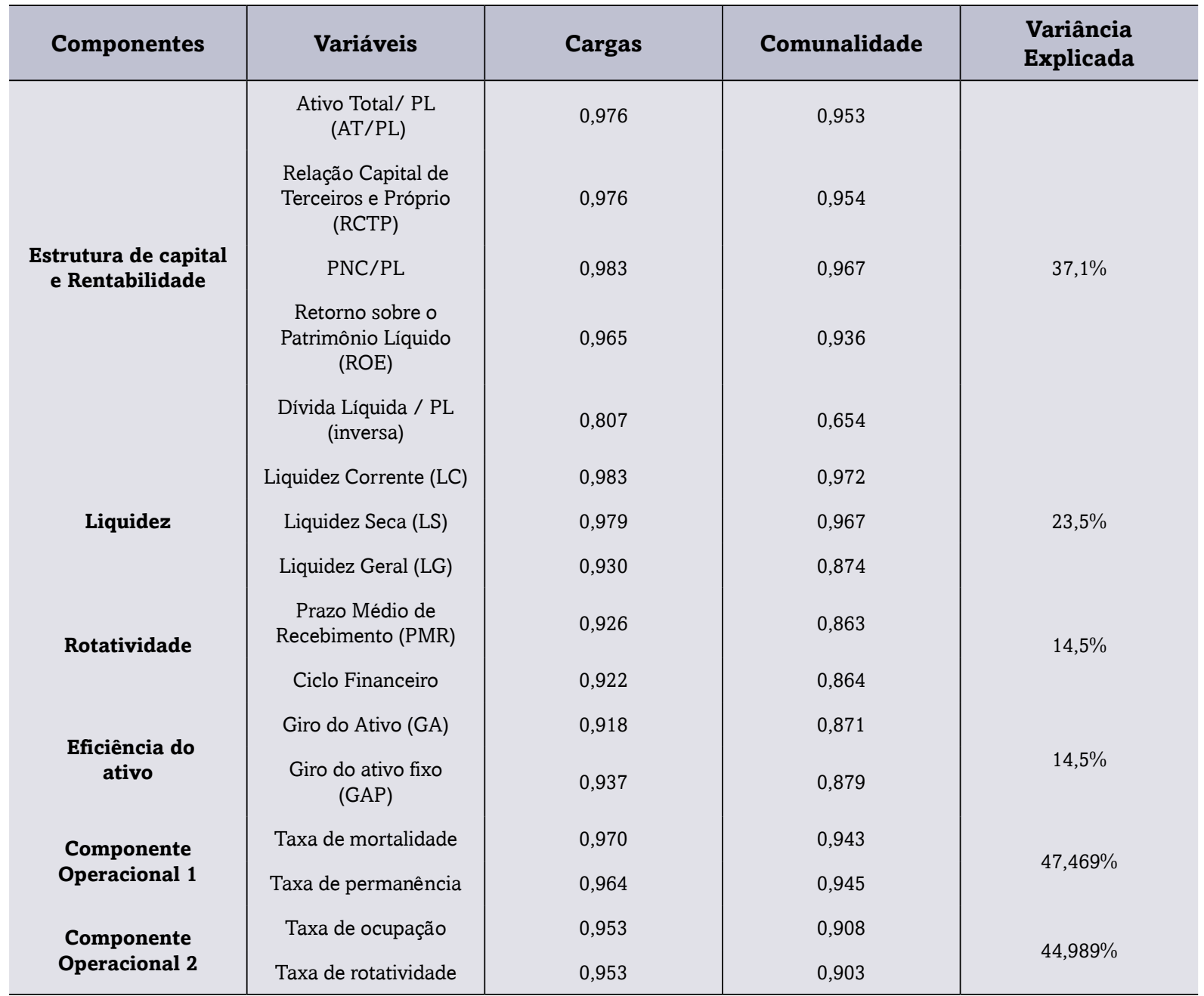

Fonte: Elaborada pelos autores.

Foram extraídos quatro componentes principais das variáveis econômico-financeiras. Nota-se que os componentes principais se formaram considerando lógica semelhante à adotada para a classificação dos indicadores econômico-financeiros. Assim, o primeiro componente contempla métricas de estrutura de capital e rentabilidade das organizações hospitalares da amostra e corresponde a $37,1 \%$ da variância explicada. O segundo componente, composto pelos indicadores de liquidez, explica 23,5\% da variância da ACP. O terceiro componente contemplou os indicadores de rotatividade e possui variância explicada de $14,5 \%$. Este valor é idêntico à variância explicada do quarto componente que agregou variáveis que demonstram a eficiência dos ativos das organizações.

Procedimento idêntico ao adotado para a verificação da ACP econômico-financeira foi realizado com as variáveis operacionais. Foram extraídos dois componentes principais, o primeiro composto pelas variáveis taxa de mortalidade e taxa de permanência e o segundo formado pela taxa de ocupação e taxa de rotatividade.

\subsection{Criação do índice}

Esta subseção se destina a relatar o processo de criação de um índice de desempenho para os hospitais sem fins lucrativos. Para isso, utilizou-se a metodologia de Nagar e Basu (2002). Para a criação dos índices foram utilizados o primeiro componente principal (Krishnakumar \& Nagar, 2008) de cada um dos fatores e com vistas a mitigar a possibilidade de ausência de explicação extraiu-se apenas variáveis que apresentassem eigenvalues maiores que um. A seguir são apresentados o cálculo dos índices econômico-financeiro (IND_FIN) e operacional (IND_OP) considerando suas respectivas variáveis e a metodologia proposta por Fávero e Belfiore (2015).

$$
\text { IND_FIN: }[(F 1 * 0,371)+(F 2 * 0,235)+(F 3 * 0,145)+(F 4 * 0,145)+(F 4 * 0,145)] / 0,896
$$


Em que:

$$
\begin{gathered}
F 1=\left[\left(z f 19_{1} * 0,976\right)+\left(z f 20_{1} * 0,976\right)+\left(z f 21_{1} * 0,983\right)+\left(z f 30_{1} * 0,965\right)+\left(z f 36_{1} * 0,807\right)\right] \\
/ 4,708 \\
F 2=\left[\left(z f 1_{1} * 0,9829\right)+\left(z f 2_{1} * 0,9793\right)+\left(z f 3_{1} * 0,9303\right)\right] / 2,893 \\
F 3=\left[\left(z f 4_{1} * 0,9263\right)+\left(z f 7_{1} * 0,9223\right)\right] / 1,8449 \\
F 4=\left[\left(z f 10_{1} * 0,9177\right)+\left(z f 11_{1} * 0,9369\right)\right] / 1,855 \\
\text { IND_OP: }[(F 1 * 0,475)+(F 2 * 0,449)] 0,925
\end{gathered}
$$

Em que:

$$
\begin{aligned}
& F 1=\left[\left(\text { ZscoreTX }_{M O R T} * 0,979\right)+\left(\text { ZscoreT }_{P E R M} * 0,964\right)\right] / 1,934 \\
& F 2=\left[\left(Z \text { scoreTX }_{\text {OcUP }} * 0,953\right)+\left(\text { ZscoreT }_{\text {ROTAT }} * 0,935\right)\right] / 1,887
\end{aligned}
$$

A Tabela 7 demonstra o perfil das quatro variáveis econômico-financeiras e operacionais, bem como dos índices criados a partir dos mesmos. A variável estrutura de capital e rentabilidade possui uma amplitude muito grande, variando de - 15,70 a 2,05, confirmando os efeitos captados pelas variáveis econômico-financeiras, quando analisadas individualmente, considerando-se os grupos de indicadores. Talvez esta amplitude elevada seja resultado da provável interferência de dados atípicos já diagnosticada na estatística descritiva das variáveis anteriores. O comportamento de variação desta variável sugere que, em termos de estrutura de capital e rentabilidade, os hospitais da amostra precisam organizar suas gestões, afinal, $50 \%$ das observações estão abaixo da média.

Tabela 7 - Estatística descritiva dos fatores e índices

\begin{tabular}{c|c|c|c|c|c}
\hline & Mínimo & $\mathbf{1}^{\mathbf{0}}$ quartil & $\begin{array}{c}\mathbf{2}^{\circ} \text { quartil } \\
\text { (mediana) }\end{array}$ & $\mathbf{3}^{\circ}$ quartil & Máximo \\
\hline $\begin{array}{c}\text { Estrutura de } \\
\text { capital/Rentabilidade }\end{array}$ & $-15,70$ & 0,03 & 0,04 & 0,06 & 2,05 \\
Liquidez & $-2,03$ & $-0,58$ & $-0,30$ & 0,06 & 4,55 \\
Rotatividade & $-9,32$ & $-0,31$ & $-0,03$ & 0,27 & 5,87 \\
Eficiência do ativo & $-1,64$ & $-0,58$ & $-0,28$ & 0,22 & 4,61 \\
Permanência/Mortalidade & $-0,33$ & $-0,18$ & $-0,13$ & $-0,06$ & 12,43 \\
Eficiência Operacional & $-2,04$ & $-0,71$ & $-0,01$ & 0,46 & 2,92 \\
IND_FIN & $-6,62$ & $-0,20$ & $-0,04$ & 0,18 & 1,07 \\
IND_OP & $-1,16$ & $-0,42$ & $-0,08$ & 0,21 & 6,22 \\
\hline
\end{tabular}

Fonte: Elaborada pelos autores.

O fator liquidez apresenta apenas $25 \%$ das observações no $3^{\circ}$ quartil, portanto, apenas elas se encontram acima da média. A variação das observações também é relevante e pode ter sido resultado da captação do efeito da liquidez geral abaixo do ideal, durante determinado período, que esteja influenciando o perfil deste fator. O fator de maior amplitude é o rotatividade que apresenta mínimo de 9,32 e o máximo de 5,37 . Neste fator $50 \%$ das observações são inferiores à mediana. A situação do fator eficiência do ativo é semelhante a do fator rotatividade no que tange ao seu posicionamento em relação à media. Os indicadores que captam o comportamento do fator eficiência do ativo são muito utilizados pelas organizações com o objetivo de avaliar se os ativos organizacionais estão produzindo riquezas.

O fator eficiência operacional apresenta menor amplitude dos dados do que o fator operacional permanência e mortalidade. No entanto este apresenta sinal negativo até o $3^{\circ}$ quartil, significando que $75 \%$ das observações estão abaixo da média, o que demonstra bom desempenho operacional das organizações, pois este fator capta o comportamento das taxas de mortalidade e de permanência as quais possuem a relação inversa, ou seja, quanto menor, melhor. Os índices econômico-financeiro e operacional apresentam amplitudes bastante próximas e $50 \%$ da amostra com valores abaixo da média. Isso significa que metade das observações exibe desempenho econômico-financeiro e operacional que merece atenção, tendo em vista os valores de seus índices econômico-financeiro e operacional.

A criação de um índice de desempenho que contemple métricas econômico-financeiras e operacionais pode ofertar melhorias na área da saúde como um todo, conforme defendem Silva, Bezerra, Souza e Gonçalves (2010), pois proporciona ao agente e ao principal direcionarem seus esforços, seja de cobrança (principal em relação ao agente) ou seja de 
alcance de seus interesses. Além disso, trata-se uma tentativa de confeccionar métricas específicas para a área da saúde (Macglynn \& Asch, 1998), considerando as necessidades dos stakeholders envolvidos no processo; lembrando que Boaventura (2012) destaca que a perspectiva da teoria dos stakeholders, essencialmente, possui como mote o deslocamento do desempenho econômico para o desempenho social, ampliando o espectro de análise dos shareholders para os stakeholders. Além disso, o índice pode ser utilizado como ferramenta de controle tanto do Estado quanto de possíveis doadores de recursos, uma vez que ambos demonstram interesse, pois contribuem com recursos (financeiros e técnicos) destinados aos hospitais sem fins lucrativos e, portanto, necessitam controlar o emprego de tais recursos, de modo a verificar sua utilização e o desenvolvimento das atividades a que se destinaram (Gimenes \& Leonardo, 2009, Leal \& Famá, 2007).

\section{CONSIDERAÇÕES FINAIS}

O presente estudo teve por objetivo determinar as variáveis que melhor explicam o desempenho operacional e econômico-financeiro dos hospitais sem fins lucrativos para com isso, sugerir a construção de um índice de desempenho destas instituições brasileiras. Entende-se que a principal contribuição deste estudo foi a propositura de uma métrica que congrega variáveis operacionais e econômico-financeiras, possibilitando a abertura de caminhos para tratar as organizações hospitalares contemplando suas especificidades. Defende-se também que a adoção de métricas comuns contribuem para a evolução de tais metodologias e a sofisticação das mesmas, sem que seja necessária a utilização de diversos indicadores que tornam o processo de avaliação de desempenho penoso e, na maior parte das vezes, pouco relevante para a maior parte de seus stakeholders.

O primeiro passo realizado foi identificar na literatura, as variáveis econômico-financeiras e operacionais utilizadas para avaliar os desempenhos econômico-financeiro e operacional dos hospitais. O prazo médio de estocagem, o giro dos estoques, a margem receita não operacional e o prazo médio de pagamento foram excluídas antes da extração das variáveis, por possuírem poucas observações. No tocante às variáveis econômico-financeiras, destaca-se a liquidez seca e corrente que apresentaram resultados superiores aos da liquidez geral, resultado das próprias características do negócio, que possuem investimento em máquinas e equipamentos de elevado valor, com pagamentos realizados em longo prazo. Por meio dos indicadores de rotatividade, verificou-se que o pagamento a prazo pode ser uma característica do setor. Percebe-se, pelos indicadores de lucratividade, que operacionalmente, os hospitais se mostram eficientes ao apurarem taxas crescentes de lucros. Por fim, pelos indicadores operacionais, observou-se que houve, ao longo dos anos, decréscimo no número de leitos disponíveis e na taxa de mortalidade, bem como elevação do número de profissionais por leito.

No intuito de determinar quais variáveis melhor explicariam o desempenho operacional e econômico-financeiro dos hospitais sem fins lucrativos, fez-se uso do procedimento estatístico ACP, que resultou em quatro variáveis econômico-financeiras (Estrutura de Capital/Rentabilidade, Liquidez, Rotatividade e Eficiência dos Ativos) e duas variáveis operacionais (Permanência/Mortalidade e Eficiência Operacional). A variável Estrutura de Capital/Rentabilidade correspondeu a 37,1\% da variância explicada, enquanto as variáveis Liquidez, Rotatividade e Eficiência dos Ativos corresponderam a 23,5\%,14,5\% e 14,5\%, respectivamente. Por outro lado, as variáveis operacionais Permanência/Mortalidade e Eficiência Operacional explicaram 47,47\% e 44,49\%, nessa ordem.

Em seguida, foi proposto um índice de desempenho para os hospitais sem fins lucrativos utilizando-se a metodologia de Nagar e Basu (2002), juntamente com as respectivos variáveis e a metodologia proposta por Fávero e Belfiore (2015). Por meio dos índices econômico-financeiro e operacional, observou-se valores abaixo da média em $50 \%$ da amostra, evidenciando que metade das observações exibe desempenho econômico-financeiro e operacional que merece atenção dos gestores dos hospitais.

Além de identificar as variáveis que melhor explicam o desempenho operacional e econômico-financeiro dos hospitais sem fins lucrativos, a contribuição desta pesquisa reside na proposta de criação de um índice de desempenho destas instituições. A criação de um índice de desempenho contribui para todos os stakeholders envolvidos com os hospitais, pois possibilita informar a todos os envolvidos a real situação vivenciada pelas organizações, além de proporcionar a verificação do desempenho das mesmas, ao longo do tempo, utilizando-se da mesma métrica, o que minimizaria a possibilidade de resultados irreais. De modo semelhante, julga-se a existência e a utilização de um índice de desempenho como importantes para os gestores das organizações hospitalares, pois possibilita a verificação do desempenho dessas, por meio de número reduzido de variáveis, portanto, viabiliza às instituições, independente do porte, monitorar sua realidade, com vistas a alcançar desempenho satisfatório.

Em síntese, a utilização de um índice de desempenho pode contribuir para o planejamento das iniciativas de saúde no Brasil, por meio da promoção da interação entre os especialistas e pesquisadores na área da saúde e os tomadores de decisão, pois tal ferramenta se apresenta como possibilidade de padronização da avaliação de desempenho dos hospitais sem fins lucrativos.

Cabe destacar que este estudo apresenta como limitação o número reduzido de hospitais integrantes da amostra e, portanto, sugere-se sua replicação com uma amostra maior. Julga-se importante a expansão de pesquisas que visem desenvolver ferramental de avaliação de desempenho específico para as organizações hospitalares, pois entende-se que são organizações que exercem papel primordial para a sociedade. Em consonância com a Fundação Osvaldo Cruz (2012), entende-se como essencial o desenvolvimento de estudos que visem quantificar os benefícios sociais decorrentes da prestação de serviços dos hospitais sem fins lucrativos à sociedade, visto que os mesmos possuem isenções fiscais e alguns são financiados por meio de recursos públicos. 


\section{REFERÊNCIAS}

ANS. Agência Nacional de Saúde Complementar. (2013). Média de permanência geral. Ministério da Saúde. Disponível http:// www.ans.gov.br/images/stories/prestadores/E-EFI- 05.pdf.

Barbosa, D. M. S., Souza, A. A., \& Santos, T. A. (2015). Limitações da Avaliação de Desempenho de Hospitais: Uma Crítica à Utilização de Indicadores Financeiros e Operacionais. Revista FSA, 12(3).

Barnum, H., \& Kutzin, J. (1993). Public hospitals in developing countries: resource use, cost, financing. Johns Hopkins University Press.

Boaventura, J. M. G. (2012). Dominância de stakeholders em empresas brasileiras: contribuições à Teoria dos Stakeholders. 117f. Tese (Livre docência) - Universidade de São Paulo, São Paulo.

Brasil. (2004). Ministério da Saúde. A política do Ministério da Saúde para a atenção integral a usuários de álcool e outras drogas. Ministério da Saúde.

Brasil. (2009). Lei $n^{\circ}$ 12.101, de 27 de novembro de 2009. Dispõe sobre a certificação das entidades beneficentes de assistência social e dá outras providências. Diário Oficial da União. Disponível http://www.planalto.gov.br/ccivil_03/_ato2007-2010/2009/lei/ 112101.htm.

Cherchiglia, M. L., \& Dallari, S. G. (2006). Tempo de mudanças: sobrevivência de um hospital público. RAE Eletrônica, 5(2).

Correia, L. F. (2008). Um indice de governança para empresas no Brasil. 296f. Tese (Doutorado em Administração) - Universidade Federal de Minas Gerais, Belo Horizonte.

Cunha, J. A. C. D., \& Corrêa, H. L. (2013). Avaliação de desempenho organizacional: um estudo aplicado em hospitais filantrópicos. Revista de Administração de Empresas, 53(5), 485-499.

Dancey, C. P. \& Reidey, J. (2006). Estatistica sem matemática para psicologia. 3. ed. Porto Alegre: Artmed.

Erdmann, R. H., \& Moreira, F. K. (2010). A gestão de organizações de saúde: do diagnóstico à melhoria da gestão. In. XXXIV Encontro da ANPAD, 2010, Rio de Janeiro - RJ.

Faria, J. A., Azevedo, T. C., \& Oliveira, M. S. (2012). A utilização da contabilidade como ferramenta de apoio à gestão nas micro e pequenas empresas do ramo de comércio de material de construção de Feira de Santana/BA. Revista da Micro e Pequena Empresa, 6(2), 89-106.

Fávero, L. P., \& Belfiore, P. (2015). Análise de dados: técnicas multivariadas exploratórias com SPSS e Stata. Rio de Janeiro: Elsevier.

Francisco, J. R. S. (2014). Índice de governança corporativa: criação de valor e desempenho nas cooperativas de crédito. Tese (Doutorado em Administração) - Universidade Federal de Minas Gerais, Belo Horizonte/MG, $301 \mathrm{f}$.

FUNDAÇÃO OSWALDO CRUZ. (2012). A saúde no Brasil em 2030: diretrizes para a prospecção estratégica do sistema de saúde brasileiro. Rio de Janeiro: Fiocruz/Ipea/Ministério da Saúde/Secretaria de Assuntos Estratégicos da Presidência da República.

Gimenes, E. R., \& Leonardo, V. S. (2009). Transparência na prestação de contas por entidades do Terceiro Setor: obrigação fiscal e social. Anais...XII Convenção de Contabilidade do Rio Grande do Sul. Bento Gonçalves, Setembro, 2009.

Guerra, M., \& de Souza, A. A. (2011). Análise de desempenho de hospitais: um estudo de 26 organizações por meio da Análise Envoltória de Dados. In II Congresso Nacional de Administração e Ciências Contábeis-AdCont 2011.

Hair, J. F., Black, W. C., Babin, B. J., Anderson, R. E., \& Tatham, R. L. (2009). Análise multivariada de dados. Bookman Editora, 688p.

Huerta, T. R., Ford, E. W., Peterson, L. T., \& Brigham, K. H. (2008). Testing the Hospital Value Proposition: An Empirical Analysis of Efficiency and Quality. Health Care Management Review, 33(4), 341-349.

Iudícibus, S. (2017). Análise de Balanços, $11^{\text {a }}$ edição. Atlas.

Kaplan, R. S., \& Norton, D.P. (2008). The execution premium. Linking strategy to operations for competitive advantage. Boston: Harvard Business School Press.

Krishnakumar, J., \& Nagar, A. L. (2008). On exact statistical properties of multidimensional indices based on principal components, factor analysis, MIMIC and structural equation models. Social Indicators Research, 86(3), 481-496.

Kubrusly, L. S. (2001). Um Procedimento para calcular índices a partir de uma base de dados multivariados. Pesquisa Operacional, 21(1), 107-117.

La Forgia, G. M., \& Couttolenc, B. F. (2009). Desempenho hospitalar no Brasil: em busca da excelencia. São Paulo: Singular.

Leal, E. A., \& Fama, R. (2007). Governança nas Organizações do Terceiro Setor: um estudo de caso. In: X SemeAd - Seminários em Administração FEA/USP, São Paulo-SP.

Lima Neto, L. (2011). Análise da situação econômico-financeira de hospitais. O Mundo da Saúde, 35(3), 270-277.

Lima, S. M. L., Portela, M. C., Uga, M. A. D., Barbosa, P. R., Gerschman, S., \& Vasconcellos, M. M. (2007). Hospitais Filantrópicos e a Operação de Planos de Saúde Próprios no Brasil. Revista de Saúde Pública, 41(1), 116-123.

Malhotra, N. K. (2006). Pesquisas de marketing: uma orientação aplicada. Tradução de Laura Bocco. 4a. ed. Porto Alegre: Bookman.

Marinho, A., Moreno, A. B., \& Cavalini, L. T. (2001). Avaliação descritiva da rede hospitalar do Sistema Único de Saúde (SUS). Texto para Discussão n. 848, Instituto de Pesquisa Econômica Aplicada - IPEA.

Marinho, A., \& Façanha, L. O. (2001). Hospitais universitários: avaliação comparativa de eficiência técnica. Economia Aplicada, $4(2), 316-49$ 
Nagar, A. L., \& Basu, S. R. (2002). Weighting socio-economic indicators of human development: a latent variable approach. In: ULLAH et al. (org.). Handbook of applied econometrics and statistical inference. New York: Marcel Dekker.

Neely, A. (2005). The Evolution of Performance Measurement Research. Development from the Last Decade and a Research Agenda for the Next. International Journal of Operations \& Production Management, 25(12), 1264-1277.

Paim, J. S., Travassos, C. M. D. R., Almeida, C. M. D., Bahia, L., \& Macinko, J. (2011). O sistema de saúde brasileiro: história, avanços e desafios. Lancet, 11-31.

Perera, S., Harrison, G., \& Poole, M. (1997). Customer-focused manufacturing strategy and the use of operations-based nonfinancial performance measures: a research note. Accounting, Organizations and Society, 22 (6), 557-572.

Ramos, M. C. D. A., Cruz, L. P. D., Kishima, V. C., Pollara, W. M., Lira, A. C. O. D., \& Couttolenc, B. F. (2015). Avaliação de desempenho de hospitais que prestam atendimento pelo sistema público de saúde, Brasil. Revista de Saúde Pública, 49, 43.

Ramos, F. M, Parizotto, E. L., Silva, A. S., Ramos, J. M., \& Bampi, G. B. (2018). Relação entre indicadores de qualidade e econômicos: um estudo em uma rede de hospitais do terceiro setor do Sul do Brasil. Cadernos Saúde Coletiva, 26(4), 453-461.

Silva, A. A. (2014). Estrutura, Análise e Interpretação das Demonstrações Contábeis, $4^{\mathrm{a}}$ edição. Atlas.

Silva, F. D. F. (2008). Análise do desempenho econômico-financeiro de seguradoras. Tese de Doutorado - Universidade Federal de Minas Gerais.

Silva, M. C.; Silva, J. D. G. \& Borges, E. F. (2015). Análise de componentes principais para elaborar índices de desempenho no setor público. Revista Brasileira de Biometria, 33(3), 291-309.

Silva, K. S. B., Bezerra, A. F. B., Souza, I. M., \& Gonçalves, R. F. (2010). Conhecimento e uso do sistema de informações sobre orçamentos públicos em saúde (SIOPS) pelos gestores municipais, Pernambuco, Brasil. Cadernos de Saúde Pública, 26(2), 373-382.

Silva, M. D. O. P., Barreto, I. G., Sousa, M. V., \& Lucena, W. G. L. (2006). Uma análise comparativa dos indicadores de desempenho de uma entidade de saúde pública de Caruaru-PE: um aplicativo estratégico do Balanced Scorecard. In Congresso USP de Iniciação Cientifica (Vol. 3).

Silva, M. Z., Sell, F. F., \& Ferla, R. (2018). Relação entre Características Organizacionais e Desempenho Econômico-Financeiro em Organizações e Saúde. Advances in Scientific and Applied Accounting, 11(1), 047-070.

Silva, M. C., Silva, J. D. G., \& Borges, E. F. (2015). Análise de componentes principais para elaborar índices de desempenho no setor público. Revista Brasileira de Biometria. São Paulo, 33(3), 291-309.

Souza, A. A., Rodrigues, L. T., Lara, C. O., Guerra, M., \& Pereira, C. M. (2009). Indicadores de desempenho econômico-financeiro para hospitais: um estudo teórico. RAHIS, (3).

Tavares, A. L., \& Silva, C. A. T. (2012). A análise financeira fundamentalista na previsão de melhores e piores alternativas de investimento. Revista Universo Contábil, 8(1), 37-52.

Vecina Neto, G., \& Malik, A. M. (2011). Gestão em saúde. Rio de Janeiro: Guanabara Koogan.

Veloso, G. G., \& Malik, A. M. (2010). Análise do desempenho econômico-financeiro de empresas de saúde. RAE eletrônica, 9(1).

Watkins, A. L. (2000). Hospital financial ratio classification patterns revisited: Upon considering nonfinancial information. Journal of Accounting and Public Policy, 19(1), 73-95.

Xavier, D. O., Souza, A. A., \& Avelar, E. A. (2019). Determinantes do Desempenho Econômico-Financeiro de Operadoras de Planos de Saúde. RAHIS-Revista de Administração Hospitalar e Inovação em Saúde, 16(1).

Zeller, T. L., Stanko, B. B., \& Cleverley, W. O. (1996). A revised classification pattern of hospital financial ratios. Journal of Accounting and Public Policy, 15(2), 161-181. 